\title{
Dynamics of water evaporation and flammability from the common "dogwood" (Cornus kousa) leaf
}

\begin{abstract}
The leaves of Cornus kousa (Dogwood) were specifically analyzed for their ability to resist burning in three different conditions: fresh, oven dried, and naturally fallen leaves. Analyzing the different surface properties of each of the leaves was of particular importance and the special attributes of the epidermis layer were determined using Optical Water Contact Angle Microscopy, Optical Microscopy and Confocal Microscopy. Water droplets were displaced onto the surface of the leaves measuring each of the contact angles over a period of time until evaporation occurred, which aided the understanding of the hydrophobic and hydrophilic properties of the leaves in different settings. Optical and Confocal Microscopy were utilized to examine the topology of the leaf epidermis, not only through a micro-analysis of the epidermal layer structures, but also through a 3D model of the different course and smooth surfaces, respectively. Flame tests were conducted to contrast the pyrolyzing rates of the leaves under the three conditions. Understanding properties specific to the Cornus kousa and more specifically the dynamics in water evaporation and flammability which is helpful for numerous applications ranging from wildfire prevention to horticulture.
\end{abstract}

Keywords: Cornus kousa, leaf structure, leaf surface properties, flammability of leaves
Volume 2 Issue 4 - 2018

\author{
Joshua Vilkas,' Pik Hoi Lam, ${ }^{2}$ Nicole \\ Jacobsen, ${ }^{3}$ Yuan Xue, ${ }^{4}$ Xianghao Zuo, ${ }^{4}$ Tae Jin \\ Kim, ${ }^{4}$ Miriam Rafailovich ${ }^{4}$ \\ 'Hebrew Academy of Nassau County High School, USA \\ ${ }^{2}$ Wilson Area High School, Easton, USA \\ ${ }^{3}$ Plainedge High School, Massapequa, USA \\ ${ }^{4}$ Department of Materials Science and Chemical Engineering, \\ Stony Brook University, USA
}

Correspondence: Miriam Rafailovich, Department of Materials Science and Chemical Engineering, Stony Brook University, 100 Nicolls Rd, Stony Brook, I I794, USA, Tel + I5I6 4589 0 I I, Email Miriam.Rafailovich@stonybrook.edu

Received: August 02, 2018 | Published: August 20, 2018

\section{Introduction}

The characterization and investigation of different properties of tree leaves are the important consideration in various scientific fields. Knowing the mechanism by which leaves burn is also critical in considering when working with leaves on varying types of shrubbery. These properties are especially important when engineering new methods to mitigate wildfires, as the physical and chemical properties of the leaf are the main promoters of burning and wildfire spread. ${ }^{1-4}$

The general biological structures that compose the leaf itself are important characterization that distinguishes different types of foliage. A leaf contains three different main layers: ${ }^{.-7}$ the upper epidermis, mesophyll, and lower epidermis. The upper epidermis is the uppermost layer of the leaf and provides protection in the form of a cuticle layer containing wax, which also assists in water conservation. Another layer of epidermal cells underneath the waxy cuticle channels the sunlight into the mesophyll layer where most of the photosynthesis occurs. Similar to the upper epidermis, the lower epidermis also contains a cuticle layer of wax and the lower epidermis cells, promoting controlled gas exchange for the sustainability of the leaf. Additionally, both the upper and lower epidermis contain structures called trichomes, which are little hair-like structures with smaller crystal formations attached. ${ }^{89}$ The mesophyll layer fits in the middle of the upper and lower epidermis layers and serves as the main locus for the production of nutrients for the leaf. Protected by the other layers and with light channeled into it, the mesophyll layer not only contains the most cells with chloroplasts, but it also contains the most water. Below the upper epidermis cells are the palisade mesophyll cells, as well as the spongy mesophyll cells and vascular cells. The palisade mesophyll layer contains closely packed cells which absorb the majority of light and produced the most energy via photosynthesis. In addition, they are transparent and further channel the sunlight into the spongy mesophyll layer. In between the spongy mesophyll cells are small, humid air pockets which allow for the gas exchange during the photosynthesis process. Additionally, long vascular cells ran along the entire mesophyll layer which contains two main components, the
Xylem and Phloem. The Xylem, a straw-like structure made of dead cells and pitted cell walls transports water and solutes from the roots to the leaves and provides significant mechanical support for the leaf. Unlike the Xylem, the Phloem is composed of living cells which transport a sap substance rich in nutrients, promoting photosynthesis and the further growth of a plant. ${ }^{10}$ Understanding how each leaf is structured is beneficial for research in leaves of specific species.

Among the top three choices for America's National Tree, according to Arbor Day Foundation, the Dogwood species placed third. The tree is native to the United States and is now considered as one of the most beautiful, flowering trees amongst America's shrubbery. ${ }^{11}$ The Dogwood species, Cornus, is divided into eleven subspecies, of which our experiment targeted the subspecies Cornus kousa. ${ }^{12,13}$ This species inhabits a vast geographical area and is commonly found in the eastern, mid-western, and southern regions of America, as well as China, Japan, and Korea. It grows well in both residential and urban settings, as well as to rural and woodland areas, and hence comes in contact with large urban and rural population groups, as well as fauna in protected natural habitats.

Numerous studies have been done on plants with leaf surfaces that were super-hydrophobic or hydrophilic, such as lotus leaves, ${ }^{14}$ but little has been done for common types of plant leaves, which have greater impact on the general population. Weiss ${ }^{15}$ has investigated the contact angle of water droplets on leaves in relation to water potential. However, wax less bean plant leaves such as Phaseolus vulgaris L. and Glycine max L. Merrill were used, with alfalfa (Medicago sativa L. ) being the only relatively waxy plant leaf used. Although the author claimed that it was probably to assume that the waxy surface of the alfalfa leaf exerted control on the water droplet at leaf surface, it was left without further investigation.

Thus, our investigation focused on the properties of fresh leaves, vacuum oven dried leaves, and dry leaves that had naturally abscised from Cornus Kousa (Dogwood). Previous work was performed mostly on leaf extractions, where results from the front and back surfaces were averaged. Hence information critical to the development of 
flame retardant spray was lost. Here we demonstrate the critical differences between the front and back of the leaf in terms of water retention and hydrophobicity, when the leaf is fresh, oven dried, of naturally decayed. In order to assess the mechanism in which each type of leaf burning, a flame test was performed on all three types of leaves (this part of the investigation was especially applicable when attempting to explore wildfire behavior). Measurement on the contact angle of water droplets on the leaves over time was conducted in order to analyze the absorption and evaporation of liquids by the leaf, as well as the hydrophobic and hydrophilic surface properties of the leaf. The mass of the leaf before and after drying in a vacuum oven was collected to assess the quantity of water the leaf contained. An analysis of the surface and physical composition of the leaf on the micro-level was created in order to accurately draw conclusions from our quantitative data.

\section{Methods}

\section{Classification of leaves and selection}

Three types of Natural Cornus kousa (Dogwood) leaves were used for measurements and comparison: fresh leaves, oven dried leaves and fallen leaves (naturally dried leaves). The fresh and fallen leaves were randomly picked. Oven dried leaves were made by putting fresh leaves in CIT Alcatel 2004A Vacuum Oven for 25 minutes at $90^{\circ} \mathrm{C}$ and 400 mTorr.

\section{Monitoring of evaporation in three groups}

A droplet of deionized water was displaced onto the front and back exteriors of each fresh, oven dried, and fallen leaves. The leaves were then placed on a KSV Optical Contact Angle Meter and kept on the goniometer until the droplet was completely evaporated. The left and right angles of the droplet as it evaporated on the leaf's surface were monitored every 3 minutes. The measurement stopped when the instrument could not continue to accurately perform measurements. The process was repeated for the front and back sides of each leaf.

\section{Micro-level examination of the surface of three groups}

A VK-X250K Keyence 3D Laser Microscope was used to acquire optical and 3D mapping images of leaf surfaces of fresh, oven dried, and fallen leaves. Images were taken on both front and back side of three types of leaves at $10 \mathrm{x}$ and $20 \mathrm{x}$ magnification.

\section{Flame test}

Three fresh, three oven dried, three fallen leaves were held under flame torch with propane fuel using a $2-3 \mathrm{~cm}$ flame for 5 seconds. Then, flame was removed and time needed for fire to extinguish was recorded.

\section{Fourier-transform infrared spectroscopy (FTIR)}

FTIR images were taken on both front and back side of fresh, oven dried and fallen leaves with a Perkin Elmer Frontier FT-IR spectrometer. An attenuated total reflection accessory was also utilized. Each spectra was averaged from 32 scans.

\section{Statistical analysis}

The results represent testing on at least 5 leaves in each category from which the standard deviations were computed. The significance levels, as denominated by the $\mathrm{p}$ values were computed using a two tailed students t-test. Chi square analysis was used to fit the volumetric and contact angle changes of droplets to linear functional forms and deduce the errors associated with the rate of change. .

\section{Results and discussion}

\section{Evaporation of water droplet on the front and back surfaces of leaves}

To investigate the surface property of the leaf surface, the contact angle between the water droplet and leaf surface were taken with three minute intervals until the water droplet evaporated. Figure 1 showed the contact angle measurements of this drying process. Each of the images was produced by overlapping the image taken at the beginning, middle and end of the tests, in which the most transparent circle corresponded to the beginning, and the darkest small circle corresponded to the end. The yellow lines indicated that the height and radius of the DI water droplet through the drying process. Figure 2 represents a graphical analysis of the drying processes shown in Figure 1. The line of best fit was found for each of the data series, but the line of best fit had to be split in two for the Oven-Dried data series as it was an exponential function, not linear. Additional equations and information pertaining to the data can be found in the legend, as well. It was evident, after analyzing Figure 1, that on the fresh leaf and fallen leaf, the radius of the water droplet was unchanged on both the front and back side of the leaf. However, it was evident that on the fresh leaf and fallen leaf, the radius of the water droplet was unchanged on both the front and back side of the leaf. However, it was the height that decreased with the water evaporation. While on the oven dried leaf, both radius and height were decreasing with water evaporation. This "pinned" effect of the water regulates the water intake more effectively than the front of the fresh leaf indicated that this was a facet of the nature of the Cornus kousa. Thus, when analyzing the contact angle results of the oven dried leaves, it was important to identify the process through which it went through. Before going into the vacuum oven, it too was a fresh leaf and likely shared the same properties as described earlier of the fresh leaf. However, being exposed to the vacuum and extreme heat environment, the leaf was likely to be damaged in the waxy cuticle layer. As seen in Table 1, the decreasing rate of water droplet volume was the same for both the front and back leaves of the oven dried leaf, indicating that when the drying process took place in a controlled environment, the waxy cuticle layer decayed evenly across both surfaces. Contrasting this to the random environmental nature of the decaying leaves that were naturally dried, it was evident why the front and back sides had such significant disparities.

From the contact angle measurements, it was notable that the front side of the fresh leaf is hydrophilic while the back side was more hydrophobic. Both the front and back side of the fallen leaf were hydrophilic. Given that these two types of leaves were the two naturally occurring types, this discovery of surface energy performance proved that the development of a water based flame retardant spray for preventing wildfire spread was very promising. Based on the results, the solution could easily wet and be absorbed by the front side of fresh leaves, as well as both sides of the fallen leaves. Droplet on a fresh leaf was due to the waxy coating on the upper and lower epidermis of the leaf. Since the fallen leaf had a similar "pinned" effect, it was likely that the waxy layer was also present on a leaf undergoing decomposition.

It was important to note observations comparing the results as a group. Though the water droplet on the oven dried leaf presented a 
hydrophobic property of the surface, it differed from the "pinned" effect of the water droplets on the fresh and fallen leaves. ${ }^{16}$ This likely meant that there were different thicknesses of the waxy cuticle layer present on each of the leaves. This theory was a possible explanation for why the fallen leaf had a hydrophilic water contact angle as well as an increasingly larger average rate of water droplet evaporation. In other words, as the fallen leaf was decomposing, it was no longer replenishing its waxy cuticle layer and was therefore not able to control the amount of water intake as do the fresh and oven dried leaves. On the other hand, the fresh leaf had just been picked from the tree less than 24 hours prior and simulated a highly accurate representation of a water contact angle of a leaf still attached to its tree. Therefore, the fact that the back side of the fresh leaf was more hydrophobic and able to

(A)

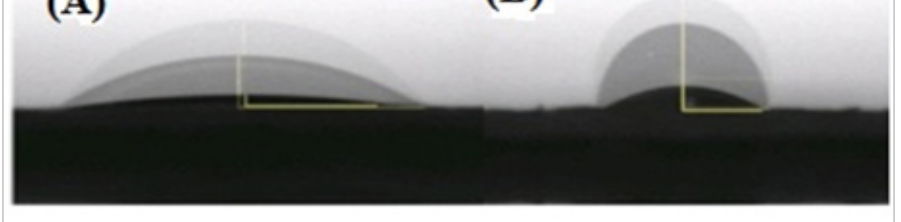

(C)

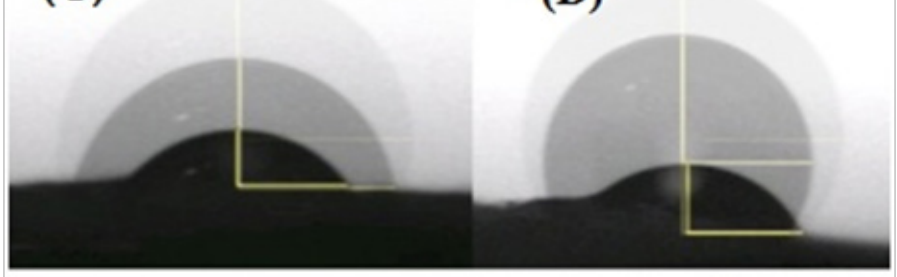

(E)

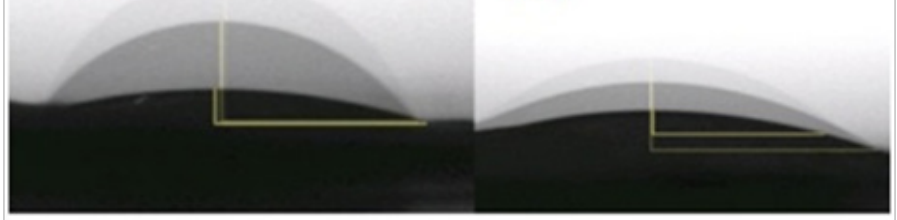

Figure I Contact angle measurements of DI water drying process on the leaf surfaces: (A) and (B) were the front and back sides of fresh leaf; (C) and (D) were front and back sides of oven dried leaf; $(E)$ and $(F)$ were front and back side of fallen leaf. Yellow lines indicated the height and radius of the DI water droplet.

\section{Mass of water loss of oven-drying}

Five leaf samples with a variety of leaf sizes were used to assess the amount of water in a standard leaf, the average weight of leaf before and after drying were shown in Table 3, and the standard deviations were calculated and shown in Figure 3A. However, it was found that, on average, the leaves were composed of $68.28 \%$ water. The water content changing could also be seen from the FTIR spectrum. The peak at around $3400 \mathrm{~cm}^{-1}$, which corresponds to water, ${ }^{17}$ was found for all three types of leaves. The intensity of this peak was found highest on both front and back side of fresh leaf, which suggest the highest water content in fresh leaves. The oven dried leaves has similar water content on both side of leaf because it was dried in a controlled and extreme environment. As for the fallen leaf, although the front side has slightly higher water content than the back side, they were both significantly less comparing to the fresh leaf. Table 3 played a significant role in the results of 5.1 by helping to understand the surface properties of the oven dried leaf. Additionally, the amount of water loss would assist in recognizing the structures in 5.3.

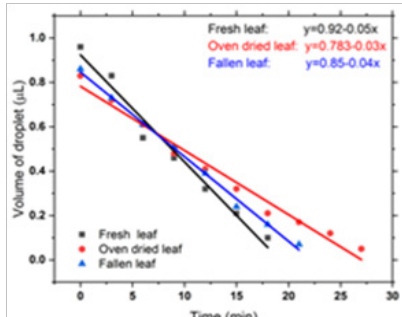

(A)

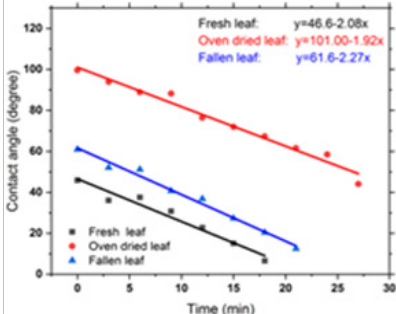

(C)

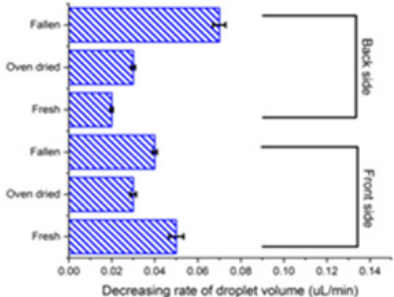

(E)

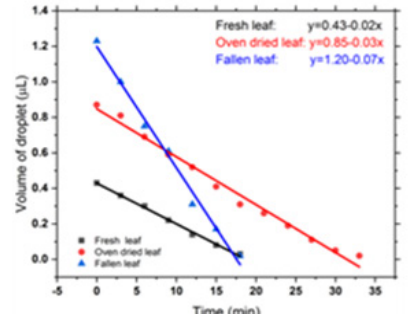

(B)

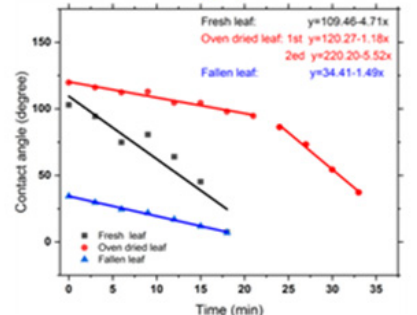

(D)

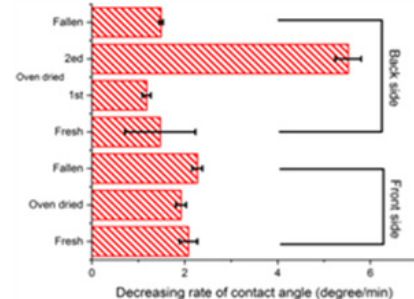

(F)
Figure 2 Change of droplet volume and contact angle over time on different leaf surfaces: (A) and (C) were the front side of leaves; $(B)$ and (D) were the back side of leaves; $(E)$ bar graph of the decreasing rate of droplet volume; $(F)$ bar graph of decreasing rate of water contact angle.

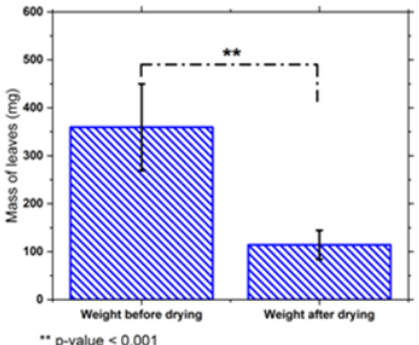

(A)

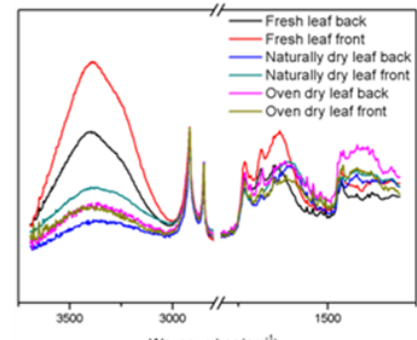

(B)
Figure 3 (A) Bar graph for weight of leaves before and after drying; (B) FTIR spectrum taken on the front and back side of fresh, oven dried and fallen leaves.

\section{Micro-level examination of the surface of three groups}

The 3D imaging of the topography of the surface of the leaves proved that there were varying levels of waxy cuticles on the epidermis of different leaves. As shown in Figure 4, the shape and surface structure of trichrome remained the same on all three types of leaves. Thus, the trichrome structure on the leaves were not affected by neither the oven drying process, nor the natural decay process. In 
both Figure 5A \& Figures $6 \mathrm{~A}$, structures that resembled bumps and spikes were waxy cuticles. ${ }^{18,19}$ Moreover, it was proved that Figure $6 \mathrm{~A}$ resembled more active structures, which correlated to the statement in 5.1 that it was in the nature for the backside of a Cornus kousa leaf to have waxier cuticles present than on its front. Additionally, it was evident that the oven drying process caused water to evaporate from the mesophyll layer, forcing the leaf to compress and form the craters seen in Figure 5C, which resembled the remaining vein structure of the xylems and the uniform coating of the remaining waxy cuticle layer. ${ }^{20}$ Furthermore, it was within line of this theory that the waxy cuticle layer of the fallen leaf was partially decomposed, and it was therefore also a viable analysis because of the partially seen textures, which resembled the waxy cuticles and the xylem crater structures in Figure 5E.
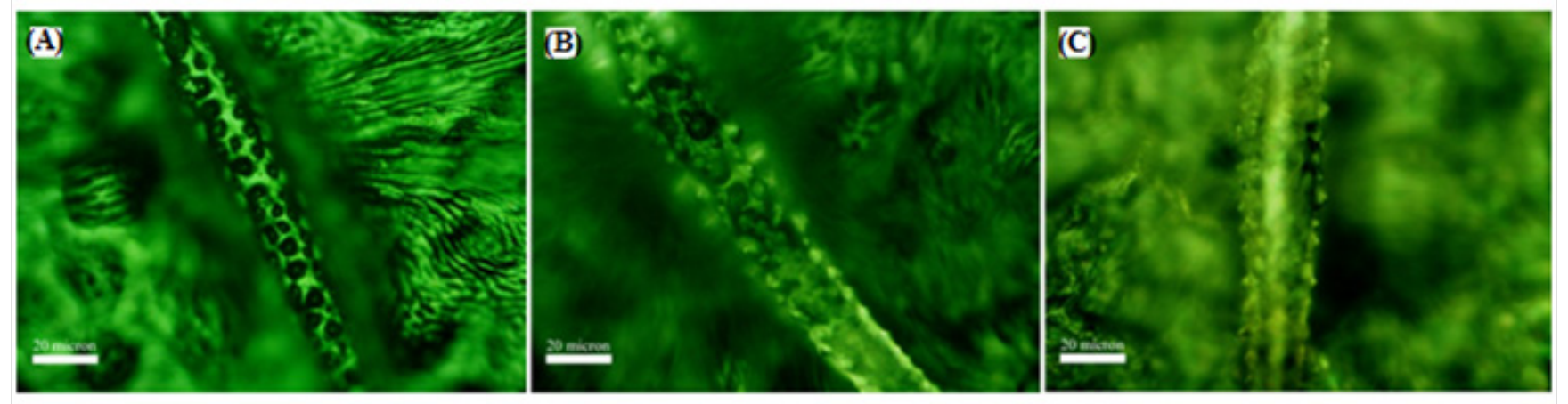

Figure 4 Optical microscope images of trichrome on three types of leaves: (A) fresh leaf; (B) oven dried leaf; and (C) fallen leaf.

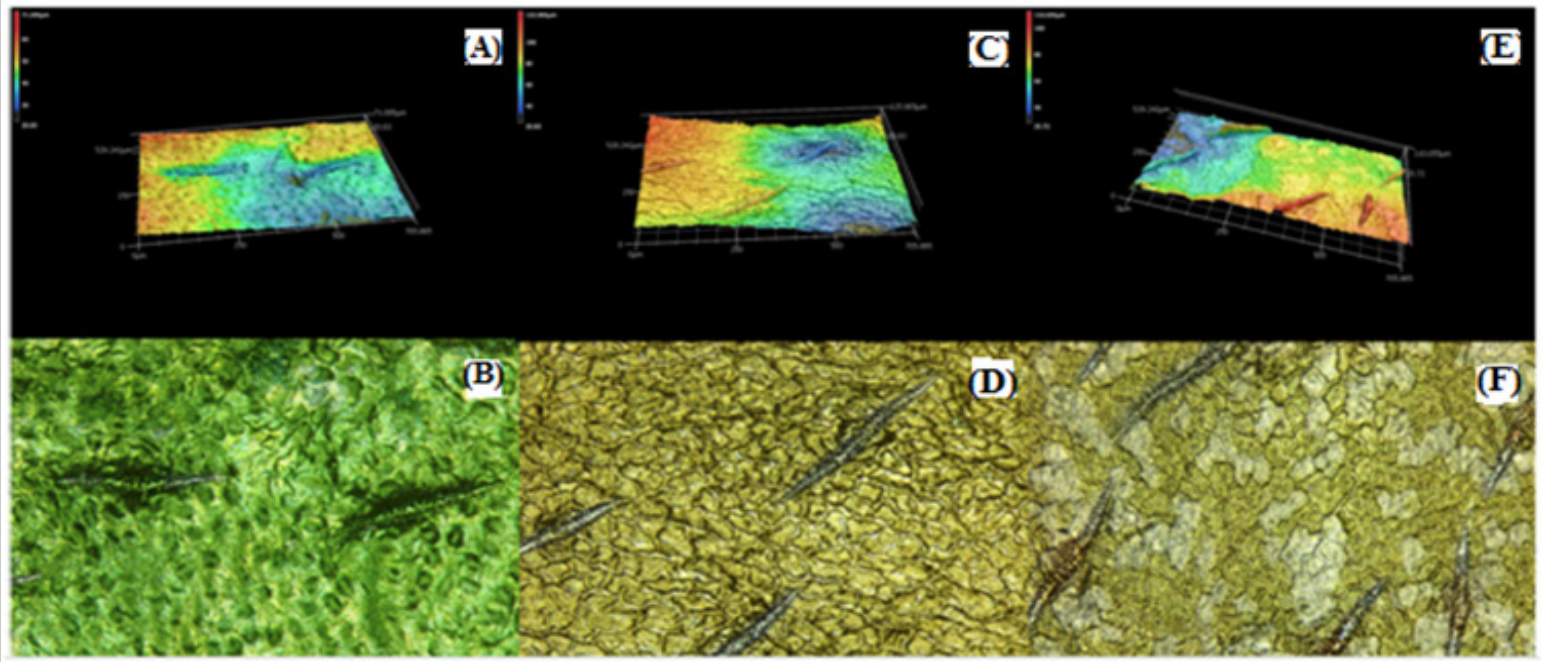

Figure 5 Optical microscope images and 3D mappings of Front side of leaf surfaces: (A) and (B) were for fresh leaf; (C) and (D) were for oven dried leaf; (E) and $(F)$ were for fallen leaf.

Table I Decreasing rate of the droplet volume and contact angle of front and back side of leaves.

\begin{tabular}{lllll}
\hline & \multicolumn{2}{l}{$\begin{array}{l}\text { Decreasing Rate of Droplet } \\
\text { volume( } \mu \mathrm{L} / \mathrm{min})\end{array}$} & \multicolumn{2}{l}{$\begin{array}{l}\text { Decreasing Rate of Contact angle } \\
\text { (degree/min) }\end{array}$} \\
\cline { 2 - 5 } & Front side & Back side & Front side & Back side \\
\hline Fresh leaf & $0.05 \pm 0.0034$ & $0.02 \pm 0.0006$ & $2.08 \pm 0.19$ & $4.71 \pm 0.76$ \\
Oven dried leaf & $0.03 \pm 0.0014$ & $0.03 \pm 0.0009$ & $1.92 \pm 0.11$ & $1.18 \pm 0.09$ \\
Naturally dried leaf & $0.04 \pm 0.0010$ & $0.07 \pm 0.0029$ & $2.27 \pm 0.11$ & $5.52 \pm 0.28$ \\
\hline
\end{tabular}

A strong indication that the leaf in Figure $6 \mathrm{~B}$ was fresh was its present green hue. It is important to note that even though a leaf might be cut from the plant, it still underwent photosynthesis within 24 hours of being cut from its source until it started decaying. ${ }^{6}$ Therefore, a fresh leaf indicated that it had been cut and used in experiments all within 24 hours. And, since the image showed a leaf that was purely green, it proved that the leaf was fresh. Because the leaf was fresh means there was also water still presented in the air pockets between the spongy mesophyll layers of the leaf, so the structures that were resembled were the spongy mesophyll cells as the laser 
light transverse did past the transparent upper epidermis and palisade mesophyll cells. The stark contrast between the fresh and oven dried leaf images could be explained first through the understanding of Table 3. Because over two thirds of the water had been lost through the process and the leaf had been in vacuum-like conditions, the oven dried leaves were compressed as the mesophyll layer had no air pockets. What could be seen left in Figure 5D was the xylem structures and the epidermis layer that had been wrapped in the outer layer. Interestingly, the heterogeneous color pattern seen in Figure 4F indicated that the leaf had undergone an enzymatic change and had already started to decompose. ${ }^{21}$ The fallen leaf image shared similar qualities to the oven dried leaf in that the xylem structures could be seen, which demonstrated that there had already been a loss of water, however, they were not as prominent. Additionally, because the oven dried leaf had a homogenous color pattern, the leaf itself did not undergo an enzymatic change and was dried uniformly because of the oven vacuum process.
Table 2 Contact angle between DI water and leaf surface.

\begin{tabular}{lll}
\hline & \multicolumn{2}{l}{$\begin{array}{l}\text { Contact angle between DI water } \\
\text { and leaf surface(degree) }\end{array}$} \\
\cline { 2 - 3 } & Front side & Back side \\
\hline Fresh leaf & 45.90 & 103.88 \\
Oven dried leaf & 85.96 & 117.92 \\
Fallen leaf & 48.78 & 34.48 \\
\hline
\end{tabular}

Table 3 Masses of leaves before and after drying in vacuum oven.

\begin{tabular}{|c|c|c|c|}
\hline & \multicolumn{2}{|c|}{ Mass of leaves (mg) } & \multirow{2}{*}{$\begin{array}{l}\text { Water loss } \\
\text { (wt.\%) }\end{array}$} \\
\hline & Before drying & After drying & \\
\hline Averaged value & 359.59 & 114.35 & 68.28 \\
\hline
\end{tabular}

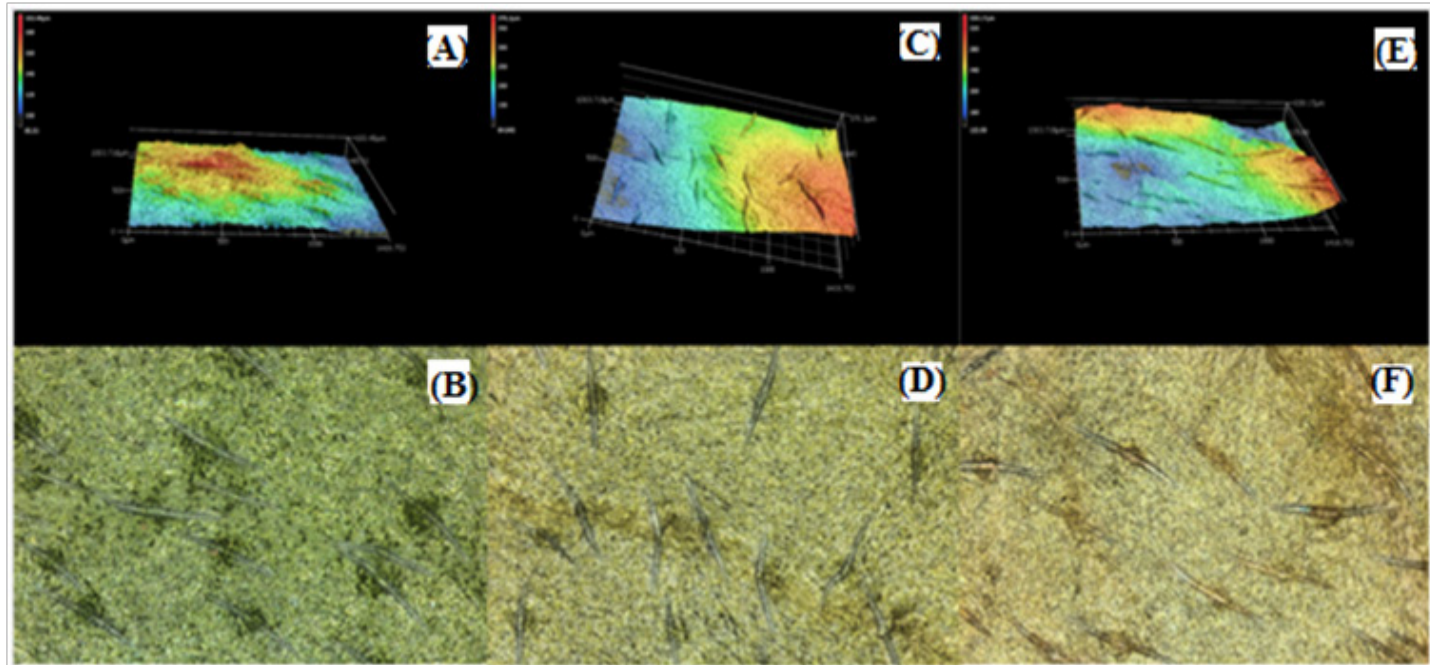

Figure 6 Optical microscope images and 3D mappings of back side of leaf surfaces: $(A)$ and (B) were for fresh leaf; (C) and (D) were for oven dried leaf; (E) and $(F)$ were for fallen leaf.

\section{Flame test}

The burning tests showed that the oven dried leaves and fallen leaves burned at the same rate, as seen in Table 4. The fresh leaves were much more resistant to the flame than the previous two. In the case of the oven dried leaf and the naturally dried leaf, 5.2 and 5.3 demonstrated that both had a loss of water. Hence, in both cases, the remaining water was shown to be lost completely in the seconds starting the burning. From the flame test results, it could be seen that water was significant in the fire resistance mechanism of the leaf. Water accounted for over $68.28 \%$ of the mass of the leaf and consequently correlated to the leaf's flame retardant properties (Figure 7).

Table 4 Time for leaves to burn out.

\begin{tabular}{llll}
\hline & Fresh leaf & $\begin{array}{l}\text { Oven dried } \\
\text { leaf }\end{array}$ & $\begin{array}{l}\text { Fallen } \\
\text { leaf }\end{array}$ \\
\hline $\begin{array}{l}\text { Time before leaf burnt out } \\
(\mathrm{sec})\end{array}$ & 45.17 & 7.08 & 6.47 \\
\hline
\end{tabular}

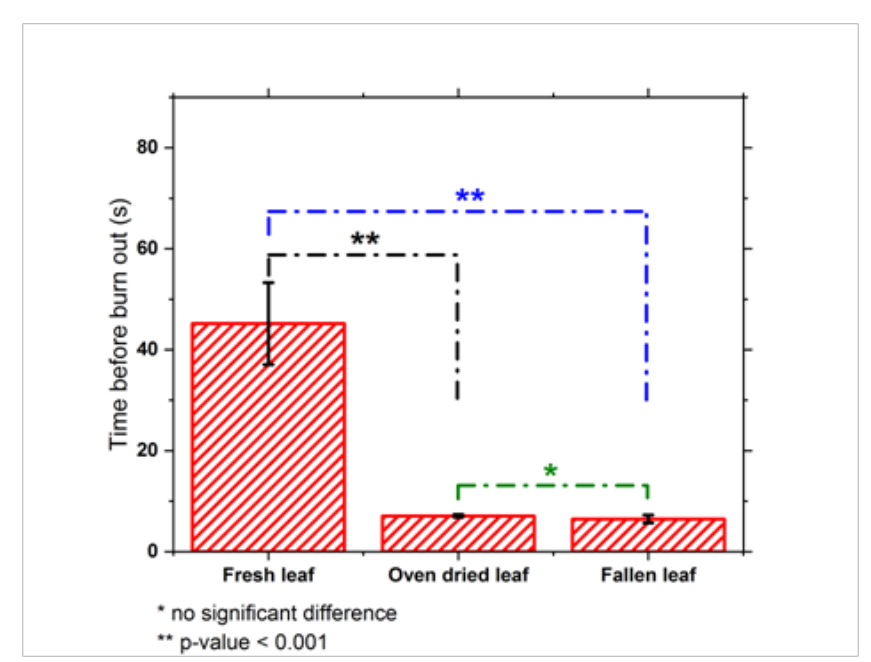

Figure 7 Bar graph showing the time before burn out for fresh, oven dried and fallen leaves. 


\section{Conclusion}

The evaporation process of water on the surface of a leaf could be labeled as "pinned", meaning as the water evaporates, and the height changed but the diameter of the droplet remained the same. From the contact angle measurements, it could be accurately concluded that the front side of the leaf was more hydrophilic, whereas the back side of the leaf was more hydrophobic due to a waxy coating. This feature was especially prominent in fresh leaves, as they were picked less than 24 hours before being examined and were still regulating water intake such as an intact leaf would on a tree. Conversely, the hot environment of a vacuum oven partially, but evenly stripped both sides of the oven-dried leaves' waxy cuticle layer. This caused the decreasing rate of droplet volume to be equivalent on both sides of the leaf. However, on the set of fallen dry leaves, the decreasing rate of droplet volume had a great disparity between the front and back sides due to environmental, random decay processes, indicating that equivalent volumetric evaporation rates on the front and back sides were not standard for all natural dried leaves. In the micro-level examination, it was found that topology models and microscopic imaging gave evidence of physical and chemical changes occurring within the leaves. In fresh leaves, water was present in the spongy mesophyll air pockets as seen in the raised bumps of its topology. These textures also indicated that it was within the nature for the Cornus kousa leaves to have different thicknesses for the waxy cuticle layers on each of its sides. Additionally, the fresh leaf had a bright green hue as it was still undergoing photosynthesis in the chloroplasts. In oven dried leaves, there was again a relatively homogenous green color, albeit less bright, and it was clearly shown in the topology imaging that there were no air pockets in the mesophyll layer, resulting in a compressed structure and the prominent presence of xylem. There was a stark contrast in the fallen leaves, as it was heterogeneously colored. The mesophyll layer was again compressed and xylem was present, but the different colors present in the images indicated that an actual enzymatic change occurred. From the flame test, we were able to conclude that dried leaves burned much faster than fresh leaves, regardless of being dried in an oven or from natural seasonal changes. The presence of the waxy cuticle layer, to which we attributed the large contact angles with water on the fresh and oven dried leaves, did not seem to affect burning the rate. Rather, the oven dried and the fallen leaves burnt at the same rapid rate, regardless of the large difference in contact angles between them. The fresh leaves, where the leaf underside contact angle was large and similar to the oven dried leaf, burned at the slowest rate. Since the major morphological difference between the three types of leaves were the swollen water laden layers in the fresh leaves vs the collapsed or absent structures in the oven dried and fallen leaves, we concluded that the presence of internal water, rather than the waxy cuticle layer, was the major factor which affected spread of fire in the leaves.

\section{Acknowledgements}

The authors would like to thank Dr. Adriana Pinkas-Sarafova, and Dr. Jonathan Sokolov among others at the Garcia Center for Polymers at Engineered Interfaces for assisting them in this experiment. We also acknowledge financial support from the National Science Foundation, the Inspire program and the Morin Foundation Trust.

\section{Conflict of interest}

Author declares that there is no conflict of interest.

\section{References}

1. Grootemaat S, Wright IJ, Van Bodegom PM, et al. Scaling up flammability from individual leaves to fuel beds. Oikos. 2017;126(10):1428-1438.

2. Giménez A, Pastor E, Zárate L, et al. Long-term forest fire retardants: A review of quality, effectiveness, application and environmental considerations. International Journal of Wildland Fire. 2004;13(1):1-15.

3. Kalabokidis KD. Effects of wildfire suppression chemicals on people and the environment - a review. Global Nest: The International Journal. 2000;2(2):129-137.

4. Larson DL, Newton WE, Anderson PJ, et al. Effects of fire retardant chemical and fire suppressant foam on shrub steppe vegetation in northern Nevada. International Journal of Wildland Fire. 1999;9(2):115-127.

5. Reich PB, Uhl C, Walters MB, et al. Leaf lifespan as a determinant of leaf structure and function among 23 Amazonian tree species. Oecologia. 1991;86(1):16-24.

6. Koike T. Leaf Structure and Photosynthetic Performance as Related to the Forest Succession of Deciduous Broad-Leaved Trees 1. Plant species biology. 1988;3(2):77-87.

7. Kikuzawa K. Leaf survival of woody plants in deciduous broad-leaved forests. 1. Tall trees. Canadian Journal of Botany. 1983;61(8):21332139.

8. Panawala L. Difference Between Upper and Lower Epidermis. 2017.

9. Redlin SC. Discula destructiva sp. nov., Cause of Dogwood Anthracnose. Mycologia. 1991;83(5):633-642.

10. Holding DR. Plant Growth Processes: Transpiration, Photosynthesis, and Respiration. USA: University of Nebraska-Lincoln; 2013.

11. Arbor Day Foundation. Dogwood: Spring Flowering Trees. USA: arborday.org; 2018.

12. Seeram NP, Schutzki R, Chandra A, et al. Characterization, quantification, and bioactivities of anthocyanins in Cornus species. Journal of Agricultural and Food Chemistry. 2002;50(9):2519-2523.

13. Tural S, Koca I. Physico-chemical and antioxidant properties of cornelian cherry fruits (Cornus mas L.) grown in Turkey. Scientia Horticulturae. 2008;116(4):362-366.

14. Barthlott W, Neinhuis C. Purity of the sacred lotus, or escape from contamination in biological surfaces. Planta. 1997;202(1):1-8.

15. Weiss A. Contact angle of water droplets in relation to leaf water potential. Agricultural and Forest Meteorology. 1988;43(3):251-259.

16. Jia H, Gogos G. Investigation of liquid droplet evaporation in subcritical and supercritical gaseous environments. Journal of Thermophysics and Heat Transfer. 1992;6(4):738-745.

17. Patel B. Extraction, characterization and application of azadirachta indica leaves for development of hygienic Lycra filaments. Advances in Chemistry. 2014;65-84.

18. Koch K, Ensikat HJ. The hydrophobic coatings of plant surfaces: Epicuticular wax crystals and their morphologies, crystallinity and molecular self-assembly. Micron. 2008;39(7):759-772.

19. Ensikat H, Boese M, Mader W, et al. Crystallinity of plant epicuticular waxes: electron and $\mathrm{X}$-ray diffraction studies. Chemistry and Physics of lipids. 2006;144(1):45-59.

20. Hansen SL, Ray PM, Karlsson AO, et al. Mechanical Properties of Plant Cell Walls Probed by Relaxation Spectra. Plant Physiology. 2011;155(1):246-258.

21. Spencer PW, Titus JS. Biochemical and Enzymatic Changes in Apple Leaf Tissue during Autumnal Senescence. Plant Physiology. 1972;49(5):746-750. 